\title{
A CORRELATIVE STUDY OF ADENOSINE DEAMINASE ACTIVITY \& T.B. IgG IN SERUM IN CASES OF TUBERCULOSIS.
}

Ajay Narang, Sanjay Kumar Nigam, Anand Mishra, Nilam Nigam

1. Assistant Professor, Department of Pathology, Rama Medical College Hospital \& Research Centre Mandhana Kanpur, U.P.

2. Associate Professor, Department of Pathology, Rama Medical College Hospital \& Research Centre Mandhana Kanpur, U.P.

3. Associate Professor, Department of Biochemistry, Rama Medical College Hospital \& Research Centre Mandhana Kanpur, U.P.

4. Associate Professor, Department of Pharmacology, Rama Medical College Hospital \& Research Centre Mandhana Kanpur, U.P.

\section{CORRESPONDING AUTHOR}

Dr. Sanjay Kumar Nigam,

House No. 308,

Rama Medical College Mandhana, Kanpur,

E-mail: sknigam@yahoo.com,

Ph: 00919411918206.

ABSTRACT: INTRODUCTION: Tuberculosis is major cause of morbidity and mortality in India as well in other parts of world. It is caused by mycobacterium tuberculosis which primarily affects lung and cause pulmonary tuberculosis. Diagnosis of tuberculosis rests upon a positive history of contact, clinical symptoms, x-ray chest, sputum positivity and AFB culture. Adenosine deaminase (ADA) is an enzyme which catalyzes the deamination of adenosine into inosine and ammonia. ADA level is found to be elevated in tuberculosis and typhoid fever where cell mediated immunity is elevated. The ADA level is significantly elevated in tuberculosis and helps to differentiate between tubercular and non tubercular diseases. The ADA level is also found to be elevated in serum and pleural fluid in patients of tubercular pleural effusion, tubercular ascitis and tubercular pericardial effusion. METHODS: Routine hemogram, Montoux test, X-ray chest, FNAC of lymph nodes, biopsy of lymph node whenever required, estimation of serum ADA level and T.B.IgG studies were performed in each case. RESULTS: In the present study a total of 45 cases were selected for the study. There are 30 cases of pulmonary tuberculosis and 15 controls. The values of serum ADA and tubercular IgG in pulmonary tubercular group are significantly higher as compared to those of controls. None of the control for ADA showed significant ratio of positivity ( $\geq 1.7$ ). One of the 15 cases showed remarkable ratio of positivity $(>1.2-1.6)$ and 14 (93.3\%) cases showed insignificant ratio of positivity. Only 2 (13.33\%) of the 15 cases showed positivity for TB IgG and rest 13 (86.66\%) were regarded negative. CONCLUSIONS: Thus it can be concluded that determination of serum adenosine deaminase levels can effectively diagnose tuberculosis with sensitivity of $96.66 \%$ and specificity of $93.33 \%$ as compared to TBIgG showing sensitivity of $90 \%$ and specificity of $86.6 \%$. Also cost of ADA estimation is remarkably less than that of tubercular IgG

KEY WORDS: Serum ADA, T.B.IgG levels, Tuberculosis

INTRODUCTION: Tuberculosis is major cause of morbidity and mortality in India as well in other parts of world. It is caused by mycobacterium tuberculosis which primarily affects lung and cause pulmonary tuberculosis. It can also affect intestine, bones, joints, lymph nodes, genitourinary system, skin and virtually every organ of the body. 
Diagnosis of tuberculosis rests upon a positive history of contact, classical symptoms, lymphocytosis on differential count, lesions in X-ray chest, sputum positivity for AFB and culture of AFB on L.J. media. But none of these except demonstration of AFB is a sure shot evidence of diagnosis. Besides this several other tests such as demonstration of tubercular antigen by Polymerase chain reaction ${ }^{1,2}$, rapid culture of tubercular bacilli by Bactec system have come forward but are costly and not available everywhere except at except at specialised centres. ${ }^{3}$

Adenosine deaminase (ADA) is an enzyme which catalyzes the deamination of adenosine into inosine and ammonia. It helps in maturation and proliferation of T cells. ADA level is found to be elevated in tuberculosis and typhoid fever where cell mediated immunity is elevated 4, 5. The ADA level is significantly elevated in tuberculosis and helps to differentiate between tubercular and non tubercular diseases. The ADA level is also found to be elevated in serum and pleural fluid in patients of tubercular pleural effusion. 6,7,8,9,10,11, tubercular ascitis 12 , 13 and tubercular pericardial effusion. ${ }^{8}$

Besides this there are several other tests which detect the antibody load against tubercular antigen in patient's serum. Enzyme linked immunosorbant assay is most frequently used and it detects the quantity and quality of circulating antibodies against tubercular antigen. Usually three antibodies are detected which include-

1. Tubercular IgA (TbIgA)

2. Tubercular IgM (TbIgM)

3. Tubercular IgG (TbIgG)

Tubercular IgA antibodies are detected in serum of some apparently healthy individuals at risk, but being the secretary antibody its detection in body fluid is of more value in patients having tubercular effusion.

Tubercular IgM is detected in initial phase of infection i.e. 1-2 months. ${ }^{14}$

Tubercular IgG is detected in patient's serum in slightly late stages when disease is properly settled (after 2 months). Its high titre is more specific as compared to tubercular IgA and IgM in patient's serum for diagnosis of active tuberculosis. ${ }^{15}$

Single detection of these antibodies alone cannot confirm the diagnosis of tuberculosis unless matched or correlated with other clinical Koch's parameters. Thus the rising antibody titres are more significant than a single high titre, but because of the cost this is practically impossible.

So the present study was undertaken to evaluate serum adenosine deaminase activity in tubercular patients.

MATERIAL AND METHODS: The present study was carried out in the department of pathology, Biochemistry, Tubercular and chest disease and Medicine Rama Medical College and Research Centre Mandhana, Kanpur.

The clinical features and detailed history were recorded in a standard proforma. Routine hemogram, Mantoux test, X-ray chest, FNAC of lymph nodes, biopsy of lymph node whenever required, estimation of serum ADA level and T.B.IgG studies were performed in each case.

Estimation of ADA activity-

We have utilized the method of GLUSEPPE GIUSTI and BRUNO GALANTI for ADA estimation. The instrument used for ADA estimation were spectrophotometer or simple 
photometer for accurate measurement between 620 and $650 \mathrm{~nm}$, water bath $37^{\circ} \mathrm{C}$, centrifuge, test tube and auto pipette.

Evaluation of T.B. IgG-

For evaluation of T.B.IgG specimen collected was patient's blood from which sera was separated and stored in a refrigerator until performance of test. Reagents used were supplied by TRANSASIA biomedical Ltd, under the name of ERBA ELISA test tuberculosis.

OBSERVATIONS: In the present study a total of 45 cases were selected for the study. There are 30 cases of pulmonary tuberculosis and 15 controls. Normal healthy individuals or patients, who were suffering from non tubercular pulmonary disease, were taken as control. The serum ADA levels and tubercular IgG of controls are shown in table I and II. Serum ADA activity and tubercular IgG of pulmonary tubercular group are shown in table III and IV.

The values in pulmonary tubercular group are significantly higher as compared to those of controls. Table V shows the comparative ratio of positivity of serum ADA and Tubercular IgG.

Table I- ADA levels in control $($ Mean $=25.83 \mathrm{U} / \mathrm{L})$.

\begin{tabular}{|l|l|l|}
\hline S.No. & ADA levels (U/L) & Ratio of positivity \\
\hline 1. & 26.65 & 1.03 \\
\hline 2. & 31.10 & 1.20 \\
\hline 3. & 30.0 & 1.16 \\
\hline 4. & 21.65 & 0.83 \\
\hline 5. & 26.65 & 1.03 \\
\hline 6. & 16.65 & 0.64 \\
\hline 7. & 18.30 & 0.70 \\
\hline 8. & 31.65 & 1.20 \\
\hline 9. & 20.0 & 0.77 \\
\hline 10. & 26.65 & 1.03 \\
\hline 11. & 28.30 & 1.09 \\
\hline 12. & 36.0 & 1.39 \\
\hline 13. & 33.30 & 1.20 \\
\hline 14. & 16.65 & 0.64 \\
\hline 15. & 23.38 & 0.90 \\
\hline
\end{tabular}

None of the control showed significant ratio of positivity ( $\geq 1.7)$. One of the 15 cases showed remarkable ratio of positivity (>1.2-1.6) and $14(93.3 \%)$ cases showed insignificant ratio of positivity.

Table - II TB IgG in controls -

\begin{tabular}{|l|l|l|l|l|}
\hline S.No. & $\begin{array}{l}\text { Observed value } \\
\text { (O.D.) }\end{array}$ & $\begin{array}{l}\text { Positive cut off } \\
\text { (O.D.) }\end{array}$ & $\begin{array}{l}\text { Ratio of } \\
\text { positivity }\end{array}$ & Interpretation \\
\hline 1. & 113 & 250 & 0.45 & - \\
\hline 2. & 226 & 250 & 0.90 & - \\
\hline 3. & 142 & 250 & 0.56 & - \\
\hline 4. & 157 & 250 & 0.67 & - \\
\hline
\end{tabular}

Journal of Evolution of Medical and Dental Sciences/Volume1/Issue5/November-2012Page-709 
ORIGINAL ARTICLE

\begin{tabular}{|l|l|l|l|l|}
\hline 5. & 440 & 250 & 1.76 & + \\
\hline 6. & 410 & 650 & 0.63 & - \\
\hline 7. & 398 & 650 & 0.61 & - \\
\hline 8. & 357 & 650 & 0.54 & - \\
\hline 9. & 401 & 650 & 0.61 & - \\
\hline 10. & 312 & 650 & 0.48 & - \\
\hline 11. & 475 & 450 & 1.05 & + \\
\hline 12. & 210 & 450 & 0.46 & - \\
\hline 13. & 317 & 500 & 0.7 & - \\
\hline 14. & 416 & 500 & 0.83 & - \\
\hline 15. & 217 & 500 & 0.43 & - \\
\hline
\end{tabular}

Only $2(13.33 \%)$ of the 15 cases showed positivity for TB IgG and rest $13(86.66 \%)$ were regarded negative.

Table III- ADA and ratio of positivity in patients of pulmonary tuberculosis.

\begin{tabular}{|c|c|c|c|}
\hline S.No. & ADA activity in serum & Cut off level & Ratio of positivity \\
\hline 1. & 58.33 & 35 & 1.66 \\
\hline 2. & 56.66 & 35 & 1.61 \\
\hline 3. & 68.33 & 35 & 1.95 \\
\hline 4. & 35.00 & 35 & 1.00 \\
\hline 5. & 70.00 & 35 & 2.00 \\
\hline 6. & 91.66 & 35 & 2.61 \\
\hline 7. & 68.33 & 35 & 1.95 \\
\hline 8. & 73.33 & 35 & 2.09 \\
\hline 9. & 65.00 & 35 & 1.85 \\
\hline 10. & 60.00 & 35 & 1.71 \\
\hline 11. & 63.33 & 35 & 1.80 \\
\hline 12. & 60.00 & 35 & 1.71 \\
\hline 13. & 61.66 & 35 & 1.76 \\
\hline 14. & 63.33 & 35 & 1.80 \\
\hline 15. & 56.66 & 35 & 1.61 \\
\hline 16. & 53.33 & 35 & 1.52 \\
\hline 17. & 68.33 & 35 & 1.95 \\
\hline 18. & 60.00 & 35 & 1.71 \\
\hline 19. & 68.33 & 35 & 1.95 \\
\hline 20. & 61.66 & 35 & 1.76 \\
\hline 21. & 60.00 & 35 & 1.71 \\
\hline 22. & 56.66 & 35 & 1.61 \\
\hline 23. & 58.33 & 35 & 1.66 \\
\hline 24. & 63.33 & 35 & 1.80 \\
\hline 25. & 61.66 & 35 & 1.76 \\
\hline 26. & 66.66 & 35 & 1.90 \\
\hline 27. & 68.33 & 35 & 1.95 \\
\hline
\end{tabular}

Journal of Evolution of Medical and Dental Sciences/Volume1/Issue5/November-2012Page-710 


\begin{tabular}{|l|l|l|l|}
\hline 28. & 63.33 & 35 & 1.80 \\
\hline 29. & 55.00 & 35 & 1.57 \\
\hline 30. & 70 & 35 & 2.00 \\
\hline
\end{tabular}

$22(73.3 \%)$ cases showed significant ratio of positivity ( $\geq 1.7)$.

$7(23.3 \%)$ cases were out of remarkable ratio of positivity (>1.2-1.6).

$1(3.3 \%)$ case showed insignificant ratio of positivity $(<1.2)$.

Table IV- TB IgG in serum of patients of pulmonary tuberculosis

\begin{tabular}{|c|c|c|c|c|}
\hline S.No. & $\begin{array}{l}\text { Observed value } \\
\text { (O.D.) }\end{array}$ & $\begin{array}{l}\text { Positive cut off } \\
\text { (O.D.) }\end{array}$ & $\begin{array}{l}\text { Ratio of } \\
\text { positivity }\end{array}$ & Interpretation \\
\hline 1. & 383 & 250 & 1.53 & ++ \\
\hline 2. & 940 & 250 & 3.76 & +++ \\
\hline 3. & 516 & 400 & 1.29 & ++ \\
\hline 4. & 207 & 400 & 0.51 & - \\
\hline 5. & 1096 & 600 & 1.82 & ++ \\
\hline 6. & 661 & 600 & 1.10 & + \\
\hline 7. & 2001 & 600 & 3.33 & +++ \\
\hline 8. & 623 & 600 & 1.03 & + \\
\hline 9. & 666 & 600 & 1.11 & + \\
\hline 10. & 696 & 650 & 1.07 & + \\
\hline 11. & 744 & 650 & 1.14 & + \\
\hline 12. & 535 & 500 & 1.07 & + \\
\hline 13. & 2046 & 500 & 4.09 & +++ \\
\hline 14. & 677 & 500 & 1.35 & ++ \\
\hline 15. & 923 & 500 & 1.84 & ++ \\
\hline 16. & 616 & 500 & 1.23 & ++ \\
\hline 17. & 814 & 500 & 1.62 & ++ \\
\hline 18. & 490 & 500 & 0.98 & - \\
\hline 19. & 894 & 500 & 1.78 & ++ \\
\hline 20. & 774 & 500 & 1.54 & ++ \\
\hline 21. & 575 & 500 & 1.15 & + \\
\hline 22. & 750 & 500 & 1.50 & ++ \\
\hline 23. & 2136 & 500 & 4.23 & +++ \\
\hline 24. & 558 & 500 & 1.11 & + \\
\hline 25. & 1231 & 500 & 2.46 & +++ \\
\hline 26. & 525 & 500 & 1.05 & + \\
\hline 27. & 878 & 500 & 1.75 & ++ \\
\hline 28. & 632 & 500 & 1.22 & ++ \\
\hline 29. & 319 & 500 & 0.63 & - \\
\hline 30. & 714 & 400 & 1.78 & ++ \\
\hline
\end{tabular}


Of these total 30 cases, 2 were clearly negative (false negative) and one was borderline negative $(+++->2)$ times positive cut off, $++->1.2-2.0$ times the positive cut off, $+->1.0-1.2$ times the positive cut off, $-<1$ times the positive cut off.

Table V-Comparison of ratio of positivity of ADA and Tubercular IgG in pulmonary tuberculosis.

\begin{tabular}{|c|c|c|c|}
\hline S.No. & Ratio of positivity of ADA & $\begin{array}{l}\text { Ratio of positivity of } \\
\text { IgG }\end{array}$ & Interpretation \\
\hline 1. & 1.66 & 1.53 & ++ \\
\hline 2. & 1.61 & 3.76 & ++ \\
\hline 3. & 1.95 & 1.29 & ++ \\
\hline 4. & 1.07 & 0.51 & +- \\
\hline 5. & 2.00 & 1.82 & ++ \\
\hline 6. & 2.61 & 1.10 & ++ \\
\hline 7. & 1.79 & 3.33 & ++ \\
\hline 8. & 2.09 & 1.03 & ++ \\
\hline 9. & 1.85 & 1.11 & ++ \\
\hline 10. & 1.71 & 1.07 & ++ \\
\hline 11. & 1.80 & 1.14 & ++ \\
\hline 12. & 1.71 & 1.07 & ++ \\
\hline 13. & 1.76 & 4.09 & ++ \\
\hline 14. & 1.80 & 1.35 & ++ \\
\hline 15. & 1.61 & 1.84 & ++ \\
\hline 16. & 1.52 & 1.23 & ++ \\
\hline 17. & 1.95 & 1.62 & ++ \\
\hline 18. & 1.71 & 0.98 & +- \\
\hline 19. & 1.95 & 1.78 & ++ \\
\hline 20. & 1.76 & 1.54 & ++ \\
\hline 21. & 1.71 & 1.15 & ++ \\
\hline 22. & 1.61 & 1.50 & ++ \\
\hline 23. & 1.66 & 4.23 & ++ \\
\hline 24. & 1.80 & 1.11 & ++ \\
\hline 25. & 1.76 & 2.46 & ++ \\
\hline 26. & 1.90 & 1.05 & ++ \\
\hline 27. & 1.95 & 1.75 & ++ \\
\hline 28. & 1.80 & 1.22 & ++ \\
\hline 29. & 1.57 & 0.63 & +- \\
\hline 30. & 2.00 & 1.78 & ++ \\
\hline
\end{tabular}

$(++)$ - Parallel rise of both the parameters.

$(+-)$ - Rise of only one parameter.

DISCUSSION: Tuberculosis continues to be a major cause of mortality and morbidity in developing countries. Although lung is the most frequent organ to be involved, inflammation of serous membranes is also very common. The definitive diagnosis is established when typical 
histological features can be demonstrated or mycobacteria can be isolated from the body fluids or sputum or on gastric lavage and various other methods such as gel electrophoresis, radiometric assay and polymerase chain reaction. It is well documented that isolation of mycobacteria and culture is very difficult and time consuming (shah et al 1990).

Recently most simple techniques which are most feasible less costly and giving quick results are now available and include demonstration of antibodies by ELISA method (humoral immune response)and ADA activity (cell mediated immune response) in serum and body fluids.

So the present study was undertaken to evaluate adenosine deaminase enzyme activity and tubercular IgG together in patients of pulmonary tuberculosis. Normal healthy individuals or patients, who were suffering from non tubercular pulmonary disease, were taken as control.

In this study the overall mean serum ADA in control was $25.83 \mathrm{U} / \mathrm{L}(16.65-36 \mathrm{U} / \mathrm{L})$. There was no significant variation in serum ADA levels in relation to age and sex in controls 16 . Only one healthy control showed increase activity of ADA in serum and may possibly be resulting from any disease involving increase cell mediated immunity e.g. typhoid ${ }^{4,5}$.

Mean serum ADA activity in patients of pulmonary tuberculosis in this study was $58.40 \mathrm{U} / \mathrm{L}$ (Table III) which was significantly elevated as compared to that of controls (Mean $25.83 \mathrm{U} / \mathrm{L}$ ). Cut off values of serum ADA > $35 \mathrm{U} / \mathrm{L}$ was diagnostic of tuberculosis with $100 \%$ sensitivity. Similar observations were given other researchers who reported higher levels of serum ADA in patients with pulmonary tuberculosis as compared to healthy controls ${ }^{16}$. The level was also significantly higher in pulmonary tuberculosis as compared to non tubercular pulmonary diseases (suppurative and malignant) and using a cut off of $33 \mathrm{U} / \mathrm{L}$. Sensitivity and specificity was claimed to be $98 \%$ and $100 \%$. Significantly higher $(\mathrm{p}<0.0005)$ serum ADA in tubercular group $(29.8 \pm 10 \mathrm{U} / \mathrm{L})$ was reported than in neoplastic group $(14.5 \pm 4.0 \mathrm{U} / \mathrm{L}){ }^{17}$. Similar data was provided by other observers who reported ADA activity of $27.38 \mathrm{U} / \mathrm{L}$ in tuberculosis as compared to malignancy (7.29) and non tubercular pulmonary diseases $(12.71)^{18}$.

Tubercular immunoglobulin IgG depicting humoral immune response was also evaluated in pulmonary tubercular patients by using ELISA method against antigen A60. In control group 2 cases (13.33\%) out of total 15 cases showed a false positivity and may be because of previous exposure to disease ${ }^{15}$. The value of optical density obtained in pulmonary tuberculosis patients are show in table IV. Three (10\%) of the total 30 cases studied showed false negative values. This could be attributed to ANERGY because of initial tubercular toxaemia or because of immunocompromised status ${ }^{19}$. The sensitivity and specificity of the test came out to be $90 \%$ and $86.6 \%$ respectively. Similar were the observations formulated by Gupta S et al 1995, who reported a specificity of $92.3 \%$ and sensitivity of $80 \%$.

Thus it can be concluded that determination of serum adenosine deaminase levels can effectively diagnose tuberculosis with sensitivity of $96.66 \%$ and specificity of $93.33 \%$ as compared to TBIgG showing sensitivity of $90 \%$ and specificity of $86.6 \%$. Also cost of ADA estimation is remarkably less than that of tubercular IgG 9. However using both tests in combination increases the specificity and sensitivity for diagnosis of tuberculosis.

\section{BIBLIOGRAPHY:}

1. 1.Brisson-Noël A, Gicquel B, Lecossier D, Lévy-Frébault V, Nassif X, Hance AJ Rapid diagnosis of tuberculosis by amplification of mycobacterial DNA in clinical samples. Lancet. 1989 Nov 4;2(8671):1069-71 
2. Tiwari V, Jain A, Verma RK. Application of enzyme amplified mycobacterial DNA detection in the diagnosis of pulmonary \& extra-pulmonary tuberculosis. Indian J Med Res. 2003 Dec;118:224-8.

3. Middlebrook G, Reggiardo Z, Tigertt WD. Automatable radiometric detection of growth of Mycobacterium tuberculosis in selective media. Am Rev Respir Dis. 1977 Jun;115(6):1066-9.

4. Galanti B, Nardiello S, Russo M, Fiorentino F. Increased lymphocyte adenosine deaminase in typhoid fever. Scand J Infect Dis. 1981;13(1):47-50

5. Mishra OP, Gupta BL, Ali Z, Nath G, Chandra L. Adenosine deaminase activity in typhoid fever. Indian Pediatr. 1994 Nov;31(11):1379-84.

6. Piras MA, Gakis C, Budroni M, Andreoni G. Adenosine deaminase activity in pleural effusions: an aid to differential diagnosis. Br Med J. 1978 Dec 2330;2(6154):1751-2.

7. Nagaraja MV, Ashokan PK, Hande HM. Adenosine deaminase in pleural effusions. J Assoc Physicians India. 1992 Mar;40(3):157-9

8. 8 Segura RM, Pascual C, Ocaña I, Martínez-Vázquez JM, Ribera E, Ruiz I, Pelegrí MD. Adenosine deaminase in body fluids: a useful diagnostic tool in tuberculosis. Clin Biochem. 1989 Apr;22(2):141-9. Gaki C.The low cost of the adenosine deaminase assay. Chest. 1996 Nov;110(5):1376-7.

9. Sharma SK, Mohan A. Adenosine deaminase in the diagnosis of tuberculosis pleural effusion. Indian J Chest Dis Allied Sci. 1996 Apr-Jun;38(2):69-71.

10. Shibagaki T, Hasegawa Y, Saito H, Yamori S, Shimokata K. Adenosine deaminase isozymes in tuberculous pleural effusion. J Lab Clin Med. 1996 Apr;127(4):34852.

11. Gupta BK, Bharat V, Bandyopadhyay D. Sensitivity, specificity, negative and positive predictive values of adenosine deaminase in patients of tubercular and non-tubercular serosal effusion in India. J Clin Med Res. 2010 May 19;2(3):121-6.

12. 13Sathar MA, Simjee AE, Coovadia YM, Soni PN, Moola SA, Insam B, Makumbi F. Ascitic fluid gamma interferon concentrations and adenosine deaminase activity in tuberculous peritonitis. Gut. 1995 Mar;36(3):419-21.

13. Gevaudan MJ, Bollet C, Charpin D, Mallet MN, De Micco P. Serological response of tuberculosis patients to antigen 60 of BCG. Eur J Epidemiol. 1992 Sep;8(5):66676.

14. Gupta S, Kumari S, Banwalikar JN, Gupta SK. Diagnostic utility of the estimation of mycobacterial Antigen A60 specific immunoglobulins IgM, IgA and IgG in the sera of cases of adult human tuberculosis. Tuber Lung Dis. 1995 Oct;76(5):41824.

15. jhamaria JP, Jenaw RK, Ludhada SK, Mathur Dk, Parihar HL, Sharma SK. Serum adenosine deaminase in the differential diagnosis of pulmonary tuberculosis and common non-tubercular respiratory diseases. Ind J Tub. 1988;35:25-7.

16. Baganha MF, Pêgo A, Lima MA, Gaspar EV, Cordeiro AR. Serum and pleural adenosine deaminase. Correlation with lymphocytic populations. Chest. 1990 Mar;97(3):605-10.

17.18 Bansal SK, Singh RP, Narang RK, Joshi LD, Bansal A, Agrawal AK. Serum adenosine deaminase in pulmonary tuberculosis, malignancy and non-tubercular respiratory diseases. Indian J Chest Dis Allied Sci. 1991 Oct-Dec;33(4):189-93.

18. Fadda G, Grillo R, Ginesu F, Santoru L, Zanetti S, Dettori G. Serodiagnosis and follow up of patients with pulmonary tuberculosis by enzyme-linked immunosorbent assay. Eur J Epidemiol. 1992 Jan;8(1):81-7. 